\title{
La dansomanie ou la passion du ballet à l'Opéra de Paris au XIX ${ }^{\mathrm{e}}$ siècle
}

\author{
Louis Bilodeau \\ Collège Ahuntsic (Montréal)
}

J'entre tout de suite dans mes idées, et je vous dis sans autre préparation que la Danse, à mon sens, ne se borne pas à être un exercice, un divertissement, un art ornemental et un jeu de société quelquefois; elle est chose sérieuse et, par certains aspects, chose très vénérable. Toute époque qui a compris le corps humain, ou qui a éprouvé, du moins, le sentiment du mystère de cette organisation, de ses ressources, de ses limites, des combinaisons d'énergie et de sensibilité qu'il contient, a cultivé, vénéré la Danse. 
Si l'on en croit Valéry, l'opéra français a fort bien compris le corps humain puisque, depuis Le Ballet comique de la Reine (1581), son histoire est indissolublement liée à celle de la danse. Lorsque Lully crée sous Louis XIV la tragédie en musique, il conçoit un «spectacle total» où chant et divertissements chorégraphiés alternent, en principe, sans nuire au déroulement de l'action. Ce modèle perdure bien longtemps après le Grand Siècle, puisque Rameau s'y conforme, de même que les plus éminents compositeurs français des Lumières. La danse en vient cependant parfois à éclipser la splendeur vocale des chanteurs, voire à représenter le principal attrait de la représentation, ce qui peut évidemment nuire à l'unité de l'œuvre. Louis de Cahusac expose bien ce problème dans l'article « divertissement » de L'Encyclopédie : « La grande règle est qu'ils [les divertissements] naissent du sujet, qu'ils fassent partie de l'action, en un mot qu'on n'y danse pas seulement pour danser. »C'est ce à quoi Gluck tendra avec ses principaux ouvrages, créés à Vienne et à Paris. Loin de s'atténuer après la Révolution, le goût très marqué des Français pour la danse va trouver de nouvelles voies d'expression tout au long du XIX ${ }^{\text {e }}$ siècle, et en particulier au Théâtre de l'Opéra. Pendant presque tout le siècle, une soirée à l'Académie Nationale (ou Impériale ou Royale) de Musique s'étire sur de nombreuses heures et comprend obligatoirement chant et danse. Cette dernière peut prendre deux formes: un ballet (parfois deux) indépendant d'un opéra assez bref (en général léger) et joué habituellement après celui-ci ou bien un divertissement intégré dans l'action d'un opéra en quatre ou cinq actes qui occupe toute la représentation. Parmi les ballets indépendants, il en est un créé à l'orée du siècle, le 14 juin 1800, dont le titre traduit bien ce que l'on observera fort longtemps 
sur la première scène lyrique parisienne : La Dansomanie. En ce jour de la bataille de Marengo, le compositeur Méhul et le chorégraphe Pierre Gardel introduisent non seulement la valse sur la scène de l'Opéra, mais font jouer le ballet qui y totalisera le plus grand nombre de représentations au XIX ${ }^{\mathrm{e}}$ siècle ${ }^{1}$. En choisissant ce titre pour leur "folie pantomime", les deux artistes annonçaient ce qui constituera un des aspects essentiels de l'opéra français pendant la majeure partie du siècle, c'est-à-dire un engouement extraordinaire pour la danse, qui, comme nous le verrons, se mariera de façon plus ou moins idéale avec le chant.

\section{Mozart dans tous ses états}

Afin de bien faire comprendre ce lien étroit entre opéra et danse, l'exemple de Mozart s'avère particulièrement éloquent. $\mathrm{Au}$ XVIII siècle, les seuls ouvrages du compositeur donnés à l'Opéra sont le modeste ballet Les Petits Riens (1778) et une adaptation très libre des Noces de Figaro ${ }^{2}$ (1793). Le 20 août 1801, l'Opéra monte La Flûte enchantée, ou plutôt Les Mystères d'Isis. Car il s'agit d'un arrangement n'ayant qu'un rapport bien lointain avec le singspiel original; cette version hautement infidèle, due aux bons soins du librettiste Morel de Chédeville et

\footnotetext{
${ }^{1} 246$ représentations selon Lajarte, p. 16. L'œuvre fut jouée pour la dernière fois le 19 avril 1826. Pour avoir accès au calendrier des représentations entre 1749 et 1989, consulter le site Chronopéra, réalisé par l'Institut de recherche sur le patrimoine musical en France (CNRS), et qui utilise le Journal de l'Opéra conservé à la Bibliothèque-Musée de l'Opéra.

2 À cette occasion, l'Opéra tenta une expérience sans lendemain : il donna pour la première fois de son histoire une œuvre non pas avec des récitatifs mais avec des passages dialogués. Pour les cinq représentations, on avait conservé le titre de la pièce de Beaumarchais, Le Mariage de Figaro.
} 
du musicien Lachnith, fut néanmoins jouée 134 fois jusqu'en 1827. C'est seulement en 1922 que l'Opéra mettra veritablement à l'affiche La Flûte enchantée. Comme il se doit, Les Mystères d'Isis comprenait d'importants divertissements (dansés sur des musiques de Haydn, de Pleyel et de Sacchini) qui suscitèrent beaucoup d'enthousiasme. En effet, Julien-Louis Geoffroy écrit dans le Journal de l'Empire : «Il n'y a point assez de danses dans les Mystères d'Isis. On sait que la danse, rivale de la musique, est un remède contre l'ennui du poëme : Gardel aurait bien fait de doubler la dose du remède, puisque l'auteur des paroles a doublé la dose de l'ennui. » (1805b, p. 4) Le critique ajoute même : « les pas de madame Gardel excitent plus d'admiration que les sons des cantatrices. » (1805c, p. 2)

C'est toujours Gardel, maître absolu du ballet de l'Opéra de 1787 à 1820, qui se charge des divertissements au moment de la création de Don Juan à Paris, en 1805. Si l'œuvre conserve son titre, elle n'en subit pas moins elle aussi de profondes mutilations effectuées par le musicien Kalkbrenner et les librettistes Thuring et Baillot, qui n'hésitent pas à déplacer l'intrigue de Séville à Naples afin de montrer l'éruption du Vésuve. Encore une fois, Geoffroy s'émerveille devant les splendeurs de la chorégraphie : «Mais parmi les agréments que ce drame rassemble, le plus flatteur est peut-être celui des ballets: le compositeur [Kalkbrenner] semble avoir voulu rivaliser avec l'auteur de la musique, et Gardel mérite le titre de Mozart de la danse. » (1805a, p. 4) Peut-être à l'issue d'une de ces représentations, Mme de Rémusat confie d'ailleurs à son mari que le chant à l'Opéra « tombe tout à fait, et on n'y va plus entendre que les jambes de Duport et de madame Gardel » (lettre du 4 décembre 1805, p. 387). 
Contrairement aux Mystères d'Isis, ce Don Juan ne survécut pas à l'Empire puisqu'il quitta l'affiche dès 1807, mais une autre version plus fidèle (traduction d'Émile Deschamps et Blaze de Bury), montée en mars 1834, allait, elle, être jouée tout au long du XIX siècle et prouver la fascination du public pour le mythe de l'éternel séducteur. Pendant tout le siècle, la scène de bal chez Don Juan comprenait un ballet. En 1834, c'est CastilBlaze (père de Blaze de Bury) qui réalisa ce travail à partir de diverses partitions de Mozart ; il avait déjà fait jouer à l'Odéon, en 1827, son Festin de pierre, dans lequel il avait inséré des dialogues de Molière entre les airs de Mozart. Pour l'Opéra, les récitatifs remplacèrent les dialogues, règle absolue de la maison, et Castil-Blaze concocta, de façon anonyme, la musique de danse qui n'eut pas l'heur de plaire à tous les critiques. Jules Janin, d'entrée de jeu, jugea complètement absurde l'idée d'interrompre la scène du bal avant le trio des masques :

Je sais bien que, puisque la scène se passe à l'Opéra, il faut un ballet à toute force; mais en quel instant arrive ce ballet, et comment vient-il ? Imaginez-vous que tout à coup le finale commencé s'arrête. Au milieu de ce bal, où vous attendez avec tant d'anxiété l'entrée des masques, la vengeance personnifiée, vous voyez entrer un chevalier maure de Grenade, dépêché en ambassade, pour demander à D. Juan la permission de laisser entrer ses braves avec leurs esclaves; et alors toute la danse du bal de Gustave entre au bal de Don Juan, et alors les chevaliers maures se mettent à danser. Je ne crois pas qu'on ait jamais rien vu de plus grotesque que ce prétendu divertissement. (1834, p. 2)

Janin ne protesta cependant pas contre l'inclusion d'un ballet au dernier tableau, avant l'apparition du commandeur. Berlioz, qui ne pouvait attaquer de front le puissant Castil-Blaze, écrivit à son ami Humbert Ferrand : «Touchez sur les ballets; ajoutez qu'ils sont d'une musique infâme (composés par Castil Blaze père !) ; vous ne pouvez en nommer l'auteur, son nom étant resté à peu près 
secret. » (1975, p. 164) À défaut de pouvoir identifier l'auteur du ballet, Berlioz, devenu critique musical au Journal des débats quelques mois plus tard, se livra néanmoins à une charge en règle contre son arrangement musical: "Malheureusement, on a cru devoir introduire dans Don Juan des airs de danse formés de lambeaux arrachés çà et là aux autres œuvres de Mozart, étendus, tronqués, disloqués et instrumentés selon la méthode qui me paraît si contraire au sens musical et aux intérêts de l'art.» (1835b, p. 1) Quoi qu'il en fût, l'Opéra redonna ces danses jusqu'en 1866, année où, à l'occasion d'une nouvelle production, la direction commanda un ballet à Auber. Prenant toujours place au même endroit, le divertissement s'intitulait «Papillons et Roses » (chorégraphie d'Arthur Saint-Léon). Lors de la dernière reprise de l'œuvre au XIXe siècle, en 1896, le ballet suscitait encore la même ferveur. À preuve, Ernest Reyer commençait son compte rendu par un long commentaire dédié à la danse : «Voilà un magnifique spectacle dans lequel le ballet, aux yeux de certains dilettantes, pourrait bien jouer le premier rôle.» Et il ajoutait que le divertissement s'impose d'autant plus que l'opéra de Mozart «dont les dimensions [...] semblaient bien mesquines ${ }^{3}$ » (1896, p. 1) à l'ancien directeur Émile Perrin est maintenant donné dans la très grande salle du nouvel Opéra de Charles Garnier.

\section{Caractéristiques du divertissement d'opéra}

À la lumière du sort réservé aux deux opéras de Mozart, on peut mieux comprendre la nature du divertissement tel que l'Opéra

\footnotetext{
${ }^{3}$ Reyer donne le détail des extraits composant le ballet: menuet de la symphonie en sol mineur (1788), allegretto varié qui termine le deuxième quatuor en ré mineur dédié à Haydn (1783), trio du menuet du quintette en mi bémol transposé en ré (1791) et Rondo alla turca de la sonate pour piano en la majeur.
} 
de Paris le conçoit au XIX ${ }^{\mathrm{e}}$ siècle. Il s'agit plus que d'un simple hors-d'œuvre, mais bien d'une partie fondamentale de toute soirée lyrique. Il peut prendre des proportions imposantes, soit une bonne demi-heure, et constituer à lui seul un acte entier, comme dans Aladin ou la lampe merveilleuse (1822) d'Isouard et Benincori, où l'on utilisa pour la première fois l'éclairage au gaz. Quoique parfois décrié par certains critiques qui lui reprochent d'interrompre l'action ou sa piètre qualité musicale, il contribue à l'éclat sans pareil des représentations de l'Opéra. Situé en général dans la deuxième moitié du spectacle, il peut revêtir, pour une partie des spectateurs, un attrait infiniment supérieur à celui du chant. Il est même parfois interchangeable. Ainsi, le divertissement de La Juive (1835) est-il abandonné en 1866 au profit de celui du Juif errant (1852) du même Halévy. Afin de redonner son ballet Aristée et les abeilles qui avait triomphé dans plusieurs villes européennes, le chorégraphe Saint-Léon n'avait pas hésité à substituer une évocation de la mythologie à un argument médiéval. Loin de regretter le ballet original, Théophile Gautier approuve le changement : «mais on a jugé à propos de le remplacer par le divertissement des Abeilles, tiré du Juif errant, qui est plus neuf, plus frais et plus dans les idées du jour. Ce n'est pas nous qui nous en plaindrons. » (1866, p. 2) Les changements de divertissement, de même que les innombrables ajouts ou suppressions qu'on leur fait subir, ne semblent pas offusquer le public, mais au contraire renouvellent son plaisir à entendre des ouvrages souvent repris depuis des dizaines d'années. Cela prouve avec éloquence combien le lien entre l'intrigue de l'opéra et les parties chorégraphiées est presque toujours extrêmement ténu, ce que déplorent d'ailleurs bon nombre de critiques tout au long du siècle. 
Une soirée sans danse est une sorte d'hérésie pour l'Opéra, à tout le moins une lacune inadmissible. $\mathrm{Si}$, au moment de la création de Lucie de Lammermoor de Donizetti en 1846, l'œuvre est donnée seule, la représentation s'achève quand même avec un divertissement de danse, «le tout terminé par une polka générale ${ }^{4}$ » (Gautier, 1846). Gautier ne semble guère sensible au caractère incongru de cet « épilogue » après la scène de la folie de Lucie et le suicide d'Edgard... Après quatre représentations, l'opéra sera d'ailleurs suivi d'un ballet ( $L a$ Péri). Amateur passionné de danse et auteur notamment de l'argument de Giselle (1841), Gautier ne peut manquer de regretter, au moment de la création de Sapho de Gounod (1851), l'absence totale de divertissement : « Est-ce par gravité personnelle, ou par égard aux scrupules solennels de M. Gounod, que M.Émile Augier n'a pas réservé de place au ballet dans son poème? Cette omission, qui attriste beaucoup l'ouvrage, déjà peu gai par lui-même, est impardonnable dans un sujet grec. » (p. 2) Tout comme pour Lucie de Lammermoor, l'œuvre de Gounod sera donnée seule quatre soirs, puis avec un ballet; pour la reprise de 1884, Gounod ajoutera un divertissement. À la fin du siècle, Rigoletto et Samson et Dalila, donnés à l'Opéra respectivement en 1885 et 1892, seront eux aussi jugés trop courts pour meubler à eux seuls toute une soirée à l'Académie Nationale de Musique. Après neuf représentations (Rigoletto) et six (Samson et Dalila), ces œuvres seront elles aussi accompagnées d'un ballet.

La direction de l'Opéra en vient ainsi, dans bien des circonstances, à ne considérer une œuvre lyrique que comme la

\footnotetext{
${ }^{4}$ Gautier ajoute : « On avait pensé que la danse s'encadrerait mal dans l'opéra de Lucie de Lammermoor à cause de sa couleur élégiaque et de l'habitude où l'on est de le voir privé de cet ornement. »
} 
première partie nécessaire d'un ballet. Par exemple, Guillaume Tell (1829), ultime chef-d'œuvre de Rossini, se réduira très souvent, avant la reprise de 1837 (débuts triomphaux de Duprez), aux premiers actes, voire seulement au deuxième, qui mettront en appétit les spectateurs pour le ballet final. D'ailleurs, est-il besoin de rappeler la désinvolture d'une large part du public qui, à cette époque, se permettait d'arriver très en retard au théâtre et de repartir avant la fin du spectacle ${ }^{5}$ ? Dans Les Grotesques de la musique, Berlioz s'insurge contre cette espèce de soumission de l'opéra à la danse : "Quand un ballet nouveau a triomphé, on taille, on rogne, on déchire, on extermine un opéra quelconque, fût-ce un chef-d'œuvre consacré par l'admiration générale, pour en faire le complément de la soirée que le ballet ne suffit pas à remplir, pour en faire un lever de rideau.» (1880, p. 209) En somme, Berlioz aurait pu faire sienne cette tirade écrite en 1725 :

Oui, je sais qu'il veut que tout danse,

Quand ce serait hors de cadence.

C'est le grand tic de l'Opéra,

Ce sont ses grâces capitales ;

On voit sur ce théâtre-là

Se trémousser jusqu'aux vestales ${ }^{6}$.

\footnotetext{
${ }^{5}$ Dans sa critique de La Fronde de Niedermeyer, Berlioz s'afflige du retard des spectateurs: "Pas plus à la première qu'à la centième représentation d'un ouvrage, pas plus à huit heures qu'à sept, les propriétaires des premières loges ne sont à leur poste. La curiosité même, ce vulgaire sentiment si puissant sur la plupart des esprits, est impuissante à les entraîner aujourd'hui. L'affiche annoncerait pour le premier acte d'un opéra nouveau un trio chanté par l'ange Gabriel, l'archange Michel et sainte Madeleine en personne, que l'affiche aurait tort, et la sainte et les deux esprits célestes chanteraient devant des loges vides et un parterre inattentif, comme de simples mortels. » (1853, p. 1).

${ }^{6}$ Lesage, Fuzelier et d'Orneval, L'Enchanteur Mirliton, opéra-comique créé à la Foire Saint-Laurent le 21 juillet 1725, musique de Jean-Claude Gillier ; l'œuvre
} 


\section{Le ballet et le grand opéra : l'époque romantique}

Réalisant à merveille les principes de la Préface de Cromwell de Victor Hugo, le grand opéra français naît à la fin des années 1820 avec La Muette de Portici (1828) d'Auber, suivie de Guillaume Tell (1829) de Rossini, Robert le Diable (1831) et Les Huguenots (1836) de Meyerbeer puis La Juive (1835) d'Halévy. La part réservée aux divertissements est certes importante dans chacune de ces œuvres, mais le cas de La Muette de Portici est particulièrement révélateur de la passion française pour la danse. Le librettiste Eugène Scribe et Auber ont réalisé là une sorte de gageure en confiant le rôle-titre d'un opéra à un personnage qui ne parle pas... mais qui danse. En effet, l'héroïne, Fenella, est un personnage interprété par une ballerine, idée audacieuse, pour ne pas dire paradoxale, qui était venue à Auber et Scribe lorsqu'ils avaient vu lors d'une soirée de gala un extrait de Deux Mots ou une nuit dans la forêt, opéra-comique de Dalayrac (1806) où évoluait une muette interprétée par la célèbre Emilia Bigottini. (voir Bilodeau, 2011, p.68) Le choix s'imposait d'autant plus que, selon Scribe, l'Opéra manquait alors de cantatrice de valeur. Certains critiques comme Castil-Blaze eurent beau se désoler du fait que « le silence de ce personnage porte le plus grand préjudice aux morceaux d'ensemble où sa voix devrait prendre part » (1828, p. 1), le succès de l'opéra fut phénoménal et le rôle de Fenella, créé par Lise Noblet, tentera les plus grandes danseuses du siècle, de Fanny Elssler à Emma Livry7. Qui plus est, le directeur

se moque de l'opéra-ballet Les Éléments de Destouches et Delalande. Cité dans Castil-Blaze, 1855, t. 2, p. 118.

${ }^{7}$ C'est d'ailleurs au cours d'une répétition de La Muette de Portici, en 1862, qu'Emma Livry, ayant passé outre à la consigne d'asperger son tutu d'un liquide non inflammable, fut victime du terrible accident qui allait lui coûter la vie. 
Lubbert félicita le chorégraphe Aumer en lui écrivant: "Les danses aussi variées qu'originales dont vous avez enrichi cet ouvrage ont été exécutées avec une perfection digne du premier théâtre de l'Europe, et les effets de scène sont devenus plus puissants parce que le corps de ballet, cette fois, n'est jamais resté étranger à l'action. » (lettre du 3 mars 1828, citée dans Wild, 1987, p. 187)

Auber et Scribe tentèrent de répéter un tel triomphe avec un opéra-ballet, Le Dieu et la Bayadère (1830), où le rôle de la bayadère était dévolu à celle qui devait bientôt devenir l'incarnation même du ballet romantique, Marie Taglioni. Or ce n'est pas dans cet ouvrage reçu sans grand enthousiasme qu'elle devait marquer les esprits, mais plutôt dans Robert le Diable de Meyerbeer, que le nouveau directeur Louis Véron monte en 1831 et qui suscite un véritable délire. La scène fameuse entre toutes du cloître de Sainte-Rosalie (IIIe acte) où, dans le décor célébrissime de Ciceri, prenait place le ballet des nonnes possédait non seulement le pouvoir de provoquer le frisson, mais aussi la qualité qui fait le plus souvent défaut aux divertissements: un lien étroit avec l'intrigue, puisque les religieuses damnées séduisent Robert pour qu'il s'empare d'un rameau magique. Ce tableau, où Meyerbeer réserve une grande importance au basson, ensorcela des générations de spectateurs et fit également les délices de Berlioz, qui y retrouvait «la plus prodigieuse inspiration de la musique dramatique moderne. » (1835a, p. 232)

Moins de quatre mois après la création de Robert le Diable, Marie Taglioni subjuguait le public par son art suprême en créant le ballet La Sylphide (1832), œuvre fondamentale du ballet romantique. Pendant la monarchie de Juillet, la danse 
connaît des années fastes : avec la technique de la pointe qui se développe, permettant à la ballerine d'interpréter les personnages les plus immatériels, les chorégraphes peuvent traduire les fantasmes d'une époque fascinée par le fantastique, les apparitions d'êtres surnaturels et les atmosphères embrumées, tout ce que Giselle (1841) et sa créatrice Carlotta Grisi réaliseront à la perfection.

C'est précisément à cette époque que les bals de l'Opéra, dont l'origine remonte à la Régence (2 janvier 1716), jouissent d'une ferveur exceptionnelle, en particulier avec l'arrivée, dans les années 1830, du chef et compositeur Philippe Musard, qui savait créer une atmosphère endiablée et arranger en quadrilles les derniers grands succès du répertoire lyrique. Dans Les Symphonies de l'hiver (1858), Jules Janin évoque avec nostalgie l'atmosphère enfiévrée qui prévalait lors de ces soirées de carnaval où le théâtre de la rue Le Peletier devenait le temple de Terpsichore :

Il fallait voir alors ce tombeau d'Opéra, se remplir de cris, de hurlements, de tempêtes, de rages! On y jouait des valses à coups de fouet, des rondes à coups de pistolet, des fandangos à coups de canon! Le cuivre était trop doux, le tambour était trop faible! On dansait la danse même des décolletées, et des montagnes en jupon court! Le parquet de la salle haletante était semé de poivre de Cayenne, et rien ne se voyait plus, làdedans, que des palpitations et des fièvres ; rien ne s'entendait plus que des hennissements et des chansons à boire ! On eût dit l'abîme en joie, et l'enfer en goguette ; et de minuit, jusqu'à six heures du matin, ça sautait, ça marchait, ça criait, ça portait le père Musard $^{8} !$ (1858, p. 291-292)

Ce n'est certes pas un hasard si l'on crée précisément à cette époque Gustave III ou le Bal masqué (1833) d'Auber et Scribe,

\footnotetext{
${ }^{8}$ Pour plus de détails, voir Jacq-Mioche et Wild, 2003, p. 88.
} 
qui apporte une nouvelle preuve, ô combien éloquente, de cette fureur pour la danse. En effet, tout l'ouvrage n'est qu'un immense prétexte pour le ballet du cinquième acte, pour lequel la direction avait consenti à de folles dépenses. Ébloui devant cette "profusion inouïe de femmes, de gaze, de velours, de grotesque, d'élégance», Janin se laisse étourdir par le mouvement général :

Un moment admirable encore, c'est le moment où tout ce monde se met à danser un galop infernal. Ils dansent tous comme un seul homme. Ils vont, ils viennent, ils se croisent, ils vont en long, en large, à droite et à gauche, dans tous les sens; c'est admirable! Le galop s'empare corps et âme de tous ces hommes et de toutes ces femmes ; et, bien qu'ils le dansent mollement, car cela va contre leurs habitudes de danser sans plier les jambes, c'est admirable! Vous ne sauriez croire à quel degré la vérité est poussée à ce bal masqué. (1833, p. 3)

Et aux esprits chagrins qui jugent sévèrement le livret de Scribe ou la partition d'Auber, Musset répond, dans son compte rendu pour la Revue des deux mondes : "Que nous importe, à nous, qui venons nous accouder sur un balcon, deux heures après dîner, que l'art soit en décadence, que la vraie musique fasse bâiller, que les poèmes de nos opéras dorment debout? [...] Il nous reste un galop, et du moment qu'on danse, qu'importe sur quel air? » (1951, p. 1000)

\section{Verdi compositeur de ballet}

Lors de son premier séjour à Paris, en 1847, Verdi ne se doutait sûrement pas qu'il mettrait en musique une adaptation du même Gustave III pour son Ballo in maschera (1859). En 1847, la carrière internationale de Verdi prend son envol : après avoir 
fait jouer I Masnadieri à Londres, il remanie I Lombardi pour l'Opéra de Paris. L'œuvre, devant impérativement être adaptée aux usages français, s'intitule désormais Jérusalem; Verdi a revu l'ensemble de la partition et a pour l'occasion composé son premier divertissement. Voilà ce que Rossini avait déjà effectué pour Maometto secondo et Mosè in Egitto, devenus respectivement Le Siège de Corinthe (1826) et Moïse (1827), œuvres annonçant le grand opéra. Comme son illustre devancier, Verdi tenta de maîtriser toutes les facettes de l'opéra à la française, mais n'y réussit pas dès cette première tentative. Avec Les Vêpres siciliennes (1855) et surtout Don Carlos (1867), commandes de l'Opéra, il parvient au sommet de son art et transcende toutes les règles du grand opéra français, sauf... celles du ballet, qui lui causera toujours de sérieux maux de tête. Il souhaitait par-dessus tout étonner le public avec un tableau imprévu comme celui du ballet du Prophète ${ }^{9}$ (1849) de Meyerbeer, où les danseurs arrivèrent chaussés de patins à roulettes. En fait, il ne parviendra qu'une seule fois, avec Aïda, à bien intégrer les danses à l'intérieur de la trame. Créée au Caire en 1871, cette dernière œuvre comprend trois courts divertissements qui n'interrompent pas la marche du drame. Lorsque vint en 1880 le temps de la création à Paris, le directeur Vaucorbeil souhaita que Verdi composât un nouveau ballet qui aurait conféré des proportions plus imposantes à l'ouvrage, ce à quoi Verdi s'opposa de façon catégorique en lui écrivant :

Quant au ballet la chose est plus grave. Je sais bien que Aïda est un opéra un peu court pour votre théâtre, mais je ne pourrais, et je ne saurais où placer un Ballet. Que voulez-vous que je vous

\footnotetext{
${ }^{9}$ Rappelons que c'est précisément pour ce tableau du Prophète que l'éclairage électrique fut utilisé pour la première fois à l'Opéra.
} 
dise, il me semble (pardonnez quelque chose à la vanité d'auteur) il me semble qu'Aïda doit être tel qu'il est, et qu'en ajoutant quelque chose on gâterait, passez-moi la phrase, l'architecture de l'ensemble. (lettre du 24 novembre 1879, citée dans Jürgensen, 1995, p. 124)

Malgré cette prise de position, Verdi consentit à ajouter 90 mesures au divertissement de la scène du triomphe, correspondant au pas des esclaves libyennes, passage que tous les théâtres jouent dorénavant.

$\mathrm{Au}$ soir de sa vie, auréolé de sa gloire de plus grand compositeur d'opéras vivant de son temps, Verdi dut néanmoins passer une dernière fois sous les fourches caudines lorsque l'Opéra monta Othello en 1894. Cette fois-ci l'œuvre, à l'origine, ne comportait aucun ballet, mais il dut en composer un pour le Palais Garnier, qu'il souhaita bref, cinq minutes 59 secondes précisa-t-il, et qui prenait place au troisième acte. Et pourtant, en 1887, quelques semaines après la création à Milan, Verdi fulminait contre cette tradition abhorrée; à son éditeur Giulio Ricordi, il écrivit que le divertissement était une concession, voire une "lâcheté ", que les compositeurs font à tort à l'Opéra et que, sur le plan artistique, c'est une « monstruosité » (lettre du 25 mars 1887, citée dans Jürgensen, 1995, p. 144). Ce ballet plaisait néanmoins à Toscanini, qui le programmait souvent dans ses concerts, et l'Opéra de Paris le maintint jusqu'en 1919.

\section{Wagner et la fin du siècle}

Richard Wagner, l'autre géant de l'opéra au XIXe siècle, subit lui aussi bien des vicissitudes quand il s'affaira à la création parisienne de Tannhäuser en 1861. D'abord jouée à Dresde en 
1845, l'œuvre suscita une véritable tempête dans la capitale française. Sans entrer dans les détails de cette affaire aux répercussions non seulement artistiques mais politiques ${ }^{10}$, relevons que Wagner était dès le départ perplexe quant aux chances de succès de l'entreprise. Ne se faisant aucune illusion sur les spectacles montés à l'Académie Impériale de Musique, il considérait que « l'opéra même n'y servait plus que de prétexte au ballet » $(1884$, p. 162). Mais comme un triomphe parisien pouvait signifier beaucoup pour lui, il accepta l'offre de l'Opéra et se résolut à ajouter un ballet à sa partition. En dépit des recommandations du directeur Alphonse Royer et du comte Waleski, ministre d'État, il refusa obstinément de placer son ballet au deuxième acte; il préféra l'enchaîner à l'ouverture, en tout début de soirée, alors que les très influents membres du JockeyClub n'étaient pas encore arrivés. Sur le plan dramatique, Wagner était parfaitement justifié en ajoutant sa bacchanale dans la grotte de Vénus, mais il contrevenait avec ostentation aux habitudes de l'institution. Victime d'une cabale d'une partie du public qui ne prit guère la peine d'écouter sa musique et qui adopta un comportement bien peu civilisé en utilisant des sifflets, Wagner retira Tannhäuser après trois représentations. Convenons que le langage musical des passages ajoutés dans ce premier tableau avait de quoi décontenancer les auditeurs; composées après l'achèvement de Tristan et Isolde, ces pages font un usage audacieux du chromatisme et constituent sans nul doute une des musiques de ballet les plus extraordinaires de tout le XIXe siècle. Si pour Isadora Duncan, qui réalisa la chorégraphie du Venusberg à Bayreuth en 1904, " cette musique renferme toute l'insatisfaction des sens, l'attente désespérée, la langueur passionnée, tout le cri

${ }^{10}$ La synthèse la plus complète se trouve chez Servières, 1895. Voir aussi Bilodeau, 2010, p. 197-199. 
du désir du monde » (1932, p. 147), le public de 1861 ne sut pas la comprendre.

Il faudra attendre trente ans pour qu'une autre œuvre de Wagner soit jouée à l'Opéra. Entre-temps, les mentalités auront beaucoup évolué, avec le wagnérisme qui fera fureur dans les années 1880 et pendant la Belle Époque. La création au Palais Garnier de Lohengrin en 1891 fit figure d'événement, en ce sens que la longueur de l'ouvrage rendait impossible la présentation d'un ballet le même soir et que l'on se garda bien d'en ajouter un à l'action. C'est pourquoi Adolphe Jullien put écrire : «Pareil succès est doublement significatif quand on réfléchit que ce long opéra ne contient pas le moindre ballet.» (1892, p. 454) Le rythme des productions wagnériennes s'accélère dans ces années, avec $L a$ Walkyrie (1893), la reprise de Tannhäuser (1895), Les Maîtres Chanteurs de Nuremberg (1897), Siegfried (1902), Tristan et Isolde (1904), Le Crépuscule des dieux (1908), L'Or du Rhin (1909) et Parsifal (1914). Un tel engouement de la part du public et des directeurs Gailhard et Bertrand se produit en même temps qu'une désaffection notoire pour la danse. Ainsi, aucun nouveau ballet ne sera créé à l'Opéra en 1891, 1892, 1894, 1895, 1896, 1898, 1899 et 1901, situation tout à fait exceptionnelle et qui concorde d'ailleurs avec une période de décadence de la danse.

On n'en continue cependant pas moins à créer des divertissements pour des ouvrages lyriques. De la production de la dernière décennie du siècle, deux opéras retiennent notre attention, de par le soin avec lequel leurs auteurs conçurent les ballets et à cause du sort peu enviable que la direction leur réserva. En 1894, Massenet et son librettiste Louis Gallet veillent à intégrer le plus harmonieusement possible le ballet du dernier acte de Thaïs, intitulé « La Tentation ». Ce divertissement illustrait 
les tourments du moine Athanaël aux prises avec son désir coupable pour la courtisane d'Alexandrie qu'il avait convertie. Malheureusement, selon Charles-Marie Widor, cette scène où les sept esprits de la tentation s'emparent de l'âme du cénobite était « un peu trop subtile pour les facultés compréhensives du gros public » (1894, p. 219), ce qui amena la direction à supprimer le ballet après la huitième représentation. Lors de la reprise de 1898, Massenet composa un nouveau divertissement qui survient au deuxième acte, devant la maison de Thaïs, à un moment cependant bien inopportun et qui suspend complètement l'action.

L'opéra Messidor (1897) d'Alfred Bruneau n'eut guère plus de chance en ce qui regarde son ballet, et ce, malgré la notoriété de son librettiste, Émile Zola. Ce dernier avait conçu un livret en prose, nouveauté quasi absolue à l'époque, répondant assez bien aux principes du naturalisme et où la danse répondait à une justification dramatique. À l'origine, les deux auteurs avaient prévu que « La Légende de l'or », ballet de près d'une demi-heure, serait placé au tout début du troisième acte afin de symboliser la lutte acharnée entre le désir du pouvoir et celui de l'amour. Mais devant la mise en scène et les tutus des danseuses qu'ils jugèrent complètement hors de propos, ils demandèrent à la direction de supprimer carrément le tableau. Plutôt que de se résoudre à cette extrémité, et malgré l'exemple célèbre de Tannhäuser, Bertrand et Gailhard préférèrent déplacer le ballet au tout début de la représentation, en une sorte de prologue incompréhensible. Il fallut attendre la reprise de 1917 pour que le ballet réintègre sa place d'origine. 
En faisant jouer Messidor à l'Opéra, Zola nous montre combien les règles de la vénérable institution ont longtemps été terriblement contraignantes, même pour les artistes les plus réputés. Quoi qu'il en fût de sa déception relative au ballet « La Légende de l'or », Zola n'en délaissa pas pour autant l'écriture de livrets d'opéras : sa toute dernière œuvre, Sylvanire ou Paris en amour, que le fidèle Bruneau devait mettre en musique, a pour héroïne une danseuse étoile de l'Opéra. Zola avait prévu l'insertion d'un divertissement chorégraphique pour une intrigue qui se déroule en grande partie à l'Opéra. Cet ultime projet d'un auteur pour qui la danse ne joue qu'un rôle épisodique dans son œuvre littéraire est révélateur de la fascination que l'Opéra et son ballet ont pu exercer, et continue toujours d'exercer d'ailleurs, dans l'imaginaire collectif. Ainsi, le Foyer de la danse est devenu au XIX ${ }^{\text {e }}$ siècle un lieu mythique, véritable Saint des Saints réservé aux danseuses, aux abonnés et à quelques rares visiteurs privilégiés. Parmi les élus, Degas a inlassablement témoigné de son obsession pour la danse en des dizaines de variations sur le thème de la ballerine. À l'extérieur du nouvel Opéra, du côté droit de la façade, La Danse déchaîne également les passions, mais négatives celles-là, lorsque, en 1869, on dévoile la sculpture de Carpeaux; la violence des réactions face à une œuvre jugée indécente est telle qu'une bouteille d'encre est lancée sur une des bacchantes. Le monde de la danse subjugue, mais doit s'en tenir à la plus grande pudeur, du moins à l'Opéra et à la fin du siècle, où les costumes laissent bien peu de place à la sensualité et où les hommes sont à peine tolérés comme partenaires des danseuses. Cela dit, le tournant du siècle représente un moment charnière à bien des égards. L'importance croissante du répertoire wagnérien fait en sorte qu'un grand nombre de soirées ne comprend plus de 
ballet indépendant ni même de divertissement. Les nouveaux ballets se font rares et, en 1903, a lieu le dernier bal de l'Opéra, qui ne connaîtra plus désormais que de sporadiques résurrections. Mais une nouvelle ère allait bientôt succéder à cette relative léthargie : en 1909, les Ballets russes de Diaghilev vont prendre d'assaut la capitale en présentant au Châtelet quelques-uns de leurs spectacles étincelants qui révolutionneront l'histoire de la danse et des arts en général. L'année suivante, ce sera au tour de l'Opéra d'accueillir sur sa scène la création de L'Oiseau de feu et de Schéhérazade ainsi que la reprise de Giselle, qui n'avait plus été donnée en ses murs depuis 1868. Inspirée par l'exemple des Fokine, Nijinski et Karsavina, la danse à l'Opéra pourra peu à peu retrouver un nouveau dynamisme, notamment grâce à Serge Lifar. Son autonomie vis-à-vis de l'art lyrique ne s'obtiendra toutefois qu'après la Seconde Guerre mondiale, au moment où la tradition des longues soirées mariant chant et danse disparaîtra graduellement au profit de spectacles plus homogènes. 


\section{Bibliographie}

BERLIOZ, Hector. (1835a), «De l'instrumentation de Robert-leDiable», Gazette musicale de Paris, 12juillet 1835, p. 229232.

-. (1835b), «Du Don Juan de Mozart», Journal des débats, 15 novembre 1835 , p. 1-2.

- (1853), «Académie impériale de musique. Première représentation de $L a$ Fronde, opéra en cinq actes, de MM. Auguste Maquet et Jules Lacroix, musique de $M$. Niedermeyer », Journal des débats, 6 mai 1853, p. 1-2.

-. (1880 [1859]), "La musique et la danse», dans Les Grotesques de la musique, Paris, Calmann-Lévy.

- (1975), Correspondance générale, sous la direction de Pierre Citron, Paris, Flammarion, t. 2.

BILODEAU, Louis. (2010), «Terpsichore à l'Opéra : la danse et le répertoire lyrique à Paris sous le Second Empire », dans Jean-Claude Yon (dir.), Les Spectacles sous le Second Empire, Paris, Armand Colin, p. 192-201.

-. (2011), « La pantomime ou le silence en musique », L'AvantScène Opéra, no 265 (Auber, La Muette de Portici), p. 68-75.

CASTIL-BLAZE. (1828), «Chronique musicale. Académie royale de musique. La Muette de Portici. - La Musique et la Danse", Journal des débats, 3 mars 1828, p.1-3.

- (1855), L'Académie Impériale de Musique, Paris, chez l'auteur. 
DUNCAN, Isadora. (1932), Ma vie, traduction de Jean Allary, Paris, Gallimard.

GAUTIER, Théophile. (1846), «Académie royale de Musique. Lucie de Lammermoor », La Presse, 23 février 1846, p. 1-2.

—. (1851), « Théâtre de la Nation. Sapho, opéra en trois actes, paroles de M.Émile Augier, musique de M. Charles Gounod", La Presse, 21 avril 1851, p. 1-2.

-. (1866), Feuilleton du Moniteur universel (reprise de La Juive), 9 juillet 1866, p. 1-3.

GEOFFROY, Julien-Louis. (1805a), «Académie impériale de musique. Première représentation de Don Juan, drame lyrique en trois actes, musique de Mozart», Journal de l'Empire, 19-20 septembre 1805, p. 1-4.

-. (1805b), "Académie impériale de musique. Les Mystères d'Isis », Journal de l'Empire, 11 novembre 1805, p. 2-4.

-. (1805c), "Académie impériale de musique. Don Juan», Journal de l'Empire, 26 décembre 1805, p. 1-2.

JACQ-MIOCHE, Sylvie et Nicole WILD. (2003), «Bals de l'Opéra », dans Joël-Marie Fauquet (dir.), Dictionnaire de la musique en France au XIXe siècle, Paris, Fayard, p. 88.

JANIN, Jules. (1833), « Théâtre de l'Opéra. Le Bal masqué, opéra en cinq actes, paroles de M. Scribe, musique de M. Auber», Journal des débats, $1^{\mathrm{er}}$ mars 1833, p. 1-3.

—. (1834), «Théâtre de l'Opéra. Le Don Juan de Mozart, opéra en cinq actes et traduit en vers par MM. Castilblaze et Émile Deschamps », Journal des débats, 17 mars 1834, p. 1-2. 
-. (1858), «Au ballet», Les Symphonies de l'hiver, Paris, Morizot, p. 273-294.

JULLIEN, Adolphe. (1892), "Richard Wagner. Lohengrin à l'Opéra de Paris », Musiciens d'aujourd'hui, Paris, Librairie de l'art, p. 443-454.

JÜRGENSEN, Knud Arne. (1995), The Verdi Ballets, Parme, Istituto nazionale di studi verdiani.

LAJARTE, Théodore de. (1876), Bibliothèque musicale $d u$ Théâtre de l'Opéra, Paris, Librairie des bibliophiles, t. 2.

MUSSET, Alfred. (1951 [1833]), « Bulletin théâtral. Compte rendu de Gustave III », Revue des deux mondes, 14 mars 1833, repris dans QEuvres complètes en prose, Paris, Gallimard, coll. «Bibliothèque de la Pléiade », p. 999-1002.

RÉMUSAT, Claire Élisabeth Gravier de Vergennes, comtesse de. (1881), Lettres de Mme de Rémusat 1804-1814, Paris, Calmann-Lévy.

REYER, Ernest. (1896), "Revue musicale. Théâtre de l'Opéra. Reprise de Don Juan », Journal des débats, 8 novembre 1896, p. 1-2.

SERVIÈRES, Georges. (1895), Tannhäuser à l'Opéra en 1861, Paris, Fischbacher.

VALÉRY, Paul. (1957 [1936]), «Philosophie de la danse», CEuvres I, Variété, «Théorie poétique et esthétique», Paris, Gallimard, coll. « Bibliothèque de la Pléiade », p. 1390-1403.

WAGNER, Richard. (1884), Souvenirs, traduction de Camille Benoit, Paris, G. Charpentier. 
WIDOR, Charles-Marie. (1894), "Thaïs», La Revue de Paris, $1^{\text {er }}$ avril 1894, p. 217-224.

WILD, Nicole. (1987), Décors et costumes du XIXe siècle à l'Opéra de Paris, t. 1, Paris, Bibliothèque Nationale.

\section{Résumé}

Héritier d'une tradition remontant à Louis XIV, l'Opéra de Paris a maintenu pendant presque tout le XIX siècle un lien consubstantiel entre les œuvres lyriques et la danse. Jusqu'à la Belle Époque, une soirée à l'Académie de Musique comprenait obligatoirement un ballet autonome ou un important divertissement prenant place au sein des opéras de Mozart, de Meyerbeer, de Verdi ou de Wagner. C'est seulement à partir de la révolution wagnérienne que cette règle disparut progressivement et que, avant le bouleversement des Ballets russes de Diaghilev au début du XXe siècle, la danse et l'opéra suivirent des voies de plus en plus indépendantes. 